\title{
Gezielte Variation des Beanspruchungskollektivs zur Verbesserung des Einlaufverhaltens im Trockenlauf
}

\author{
Thomas Klotz $^{1,2}$ iD $\cdot$ Katharina Bause ${ }^{1} \cdot$ Sascha Ott $^{1} \cdot$ Albert Albers $^{1}$ \\ Eingegangen: 15. Juni 2021 / Angenommen: 17. September 2021 / Online publiziert: 23. November 2021 \\ (c) Der/die Autor(en) 2021
}

\section{Zusammenfassung}

Präzise steuer- und regelbaren Kupplungen wird eine Schlüsselrolle in der Transformation der Automobilwirtschaft zugesprochen. Als Grundlage der präzisen Übertragung des Drehmoments sind zuverlässig gleichbleibende Reibeigenschaften der in der Kupplung verbauten Friktionspaarungen von großem Vorteil. Diese Reibeigenschaften können sich jedoch unter entsprechenden Randbedingungen, wie beispielsweise während des Einlaufs, bei erhöhten Temperaturen und bei stark erhöhten Beanspruchungen, äußerst volatil verhalten. Dieses Verhalten kann durch die Auswahl der Friktionswerkstoffe verbessert werden. Jüngste Forschungsergebnisse zeigen, dass das Erholungsverhalten nach einer Schädigung durch zum Beispiel eine Notfallbremsung ebenso wie durch das Beanspruchungskollektiv beeinflusst wird und durch dessen Variation nachweislich verbessert werden kann. Es bleibt zu zeigen, inwiefern die Variation des Beanspruchungskollektiv auch in anderen Situationen dazu beitragen kann, die gewünschten Reibeigenschaften zu erreichen.

Diese Studie dient dazu, dieses Potenzial anhand des Einlaufverhaltens trockenlaufender Friktionspaarungen mit organischen Reibbelägen aufzuzeigen. Um Beanspruchungskollektive abzuleiten, welche den Einlauf verkürzen, werden Erklärungsmodelle sowie Methoden, Ergebnisse und Erkenntnisse aus vorangegangenen Forschungsarbeiten genutzt. Die experimentellen Ergebnisse zeigen auf, dass eine Verkürzung des Einlaufs und eine Stabilisierung der Reibeigenschaften durch diese veränderten Beanspruchungskollektive erzielt werden können. Folglich ist es denkbar, in zukünftigen Anwendungen die Reibeigenschaften zu überwachen und bei Bedarf durch die Variation des Beanspruchungskollektiv gezielt zu regeln.

\section{Variation of load for the improvement of the running-in behaviour of dry-running friction pairings}

\section{Abstract}

Precisely controllable and adjustable clutches play a key role in the transformation of the automotive industry. Therefore, constant frictional properties of the built in friction pairings are beneficial. These frictional properties can be volatile under certain boundary conditions such as during run-in, at elevated temperatures and under greatly increased loads. This behavior can be improved due to the selection of the friction materials. Besides, the latest research results show that the recovery behavior after fade is also influenced by the load collective.

Its variation can demonstrably lead to an improvement inf the recovery behavior [1]. Nevertheless, tt remains to be shown to what extent the variation of the load collective can also contribute in other situations in order to achive the desired friction properties more precisely and more quickly.

Thomas Klotz

thomas.klotz@kit.edu

1 IPEK - Institut für Produktentwicklung, Karlsruhe, Deutschland

2 Karlsruher Institut für Technologie (KIT), Karlsruhe, Deutschland 
The aim of this study is to demonstrate this potential based on the run-in behavior of dry-running friction pairings with organic friction linings. In order to derive load collectives that shorten the running-in explanatory models as well as methods, results and findings from previous research work $[1,2]$ are used. The experimental results show that a shortening of the run-in and a stabilization of the friction properties can be achieved through these changes. Consequently, it is conceivable to monitor the frictional properties in future applications and, if necessary, to regulate them in a targeted manner by varying the load collective.

\section{Einleitung}

Anwendungen, welche auf reibschlüssiger Kraftübertragung basieren, stellen verschiedenste Anforderungen an das tribologische Verhalten ihrer Friktionspaarungen. Die Erfüllung dieser Anforderungen ist essenziell für die Funktionserfüllung und den Komfort dieser Anwendungen. Hierzu müssen beispielsweise die Reibungszahl, der Reibungszahlgradient und der Verschleißkoeffizient innerhalb zuvor spezifizierter Grenzwerte liegen [3]. Hierbei ist zu beachten, dass die in trockenlaufenden Kupplungen und Bremsen eingesetzten Friktionspaarungen einem sogenannten Einlaufprozess unterliegen, der erst nach einer Vielzahl gleichartiger Anfahr- beziehungsweise Bremsvorgängen beendet ist. Die Reibeigenschaften verändern sich während dieses Einlaufprozesses, sodass sich erst nach diesem ein quasistationärer Reibungszustand einstellt. Das heißt, dass sich die Reibeigenschaften bei gleicher Beanspruchung nicht mehr verändern [4]. Das Ziel des Einlaufs ist es, die Friktionspaarung auf ein konstantes und stabiles Reibungszahlniveau zu bringen [2] oder zumindest einen näherungsweisen linearen Verlauf des Reibungszahltrends zu erreichen [5]. Des Weiteren muss das dabei erzielte tribologische Verhalten nach wie vor die zu Beginn beschriebenen Anforderungen erfüllen.

Die Dauer des Einlaufs und die darin erzielten Reibeigenschaften variierten in Abhängigkeit der Beanspruchung. Musiol stellt beispielsweise fest, dass der daran beteiligte Aufbau der Reibschicht durch die Auswahl der Versuchsparameter beschleunigt oder verlangsamt werden kann [4]. Welche Mechanismen dabei im Detail wirken können, beschreibt der Stand der Forschung unter anderem anhand dieser Erklärungsmodelle:

- Zunahme der Eisenkonzentration und Oxidation der Reibschicht sowie die Schädigung und Regeneration von Volumenelementen nach Musiol [4],

- Bildung von primären und sekundäre Plateaus nach Eriksson [6],

- Wachsen von Kontaktplättchen und Verschleißdynamik nach Ostermeyer [7],

- Entstehung von Reibschichten nach Poeste [8],

- Bildung von Platten aufgrund lokaler Temperatur- und Druckunterschiede nach Cristol-Bulthé [9].
Reibungsverluste können des Weiteren durch folgende Modelle erklärt werden:

- Schmierwirkung von Harzzersetzungsprodukten nach Tanaka [10],

- Verschiebung des Bremskraftradius nach Lührsen [11],

- Wandern des mittleren Reibradius durch die Deformation der Bremsscheibe infolge der Temperatureinwirkung nach Gauger [12],

- Bildung eines Gaskissens nach Fidlin [13].

Auf Basis dieser Erklärungsmodelle und einer durch die Autoren entwickelten methodischen Vorgehensweise konnte gezeigt werden, dass es durch eine vorübergehende Variation des Beanspruchungskollektivs möglich ist, das Erholungsverhalten nachweislich zu verbessern. Durch die Anpassung der Temperatur oder auch der Gleitgeschwindigkeit in wenigen ersten Zyklen nach einer Notfallbremsung ist es möglich, die Reibeigenschaften schneller, mit geringerer Streuung oder auch mit erhöhter Reibung zu erholen [1].

Auf Basis der durchgeführten Untersuchungen ist es denkbar, dass ähnliche Verbesserungen auch hinsichtlich des Einlaufverhaltens erzielt werden können. Eine solche systematische Variation fehlt jedoch bisher. Daher ist es das Ziel dieser Arbeit, weitere Nutzungsmöglichkeiten der zum Erholungsverhalten entwickelten und gewonnenen Methoden und Erkenntnisse am Beispiel des Einlaufverhaltens trockenlaufender Friktionspaarungen aufzuzeigen.

\section{Methode zur Variation des Beanspruchungskollektivs im Einlauf}

Um das Potenzial einer Variation des Beanspruchungskollektivs im Einlauf beispielhaft aufzuzeigen, werden in dieser Arbeit vier verschiedene Varianten erzeugt und erprobt. Das Ziel ist es, eine Variante zu finden, welche bei einer gegebenen Friktionspaarung im Vergleich zum bestehenden Einlauf zu einem schnelleren und zuverlässigeren Anstieg der mittleren Reibungszahl führt. Dieser bestehende Einlauf wird als Referenz herangezogen. Ausgehend von diesem wird je einer der Beanspruchungsparameter, Gleitgeschwindigkeit oder Flächenpressung, variiert. Alle weiteren Beanspruchungsparameter wie die Temperatur und das Massenträgheitsmoment werden zunächst nicht verän- 


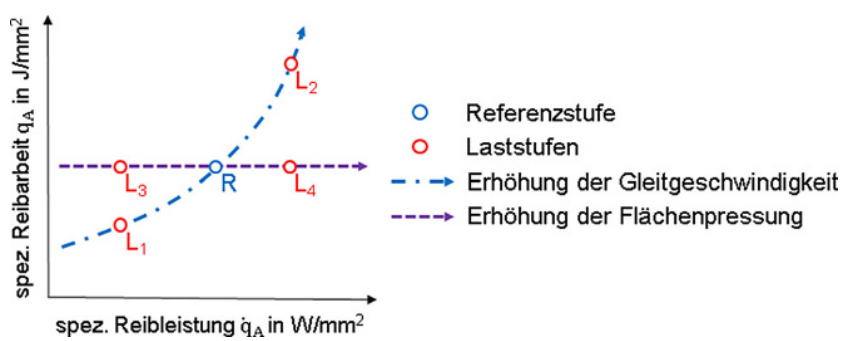

Abb. 1 Variation der Gleitgeschwindigkeit und der Flächenpressung ausgehend von der Referenzstufe

dert. Abb. 1 veranschaulicht schematisch diese Variationen im Diagramm der spezifischen Reibarbeit über der Reibleistung. Durch Absenkung und Erhöhung von Gleitgeschwindigkeit und Flächenpressung werden vier Laststufen erzeugt, welche Varianten des bestehenden Einlaufs sind. Dessen Beanspruchungskollektiv wird mittig durch die Referenzstufe markiert.

Sobald weitere Beanspruchungsparameter variiert werden sollen oder weitere Faktorstufen hinzukommen, steigt die Anzahl der Varianten schnell an. Wenn dann noch entsprechend viele Wiederholungen der einzelnen Varianten durchgeführt werden sollen, folgt daraus, dass entsprechend viel Zeit und Versuchsmaterial für die Untersuchungen notwendig ist. Es wird im Folgenden ein Kurzzeittest vorgeschlagen, welcher es ermöglicht, das Potenzial der Varianten innerhalb von kurzer Zeit und mit nur einem Exemplar der Friktionspaarung abzuschätzen. Bei diesem Kurzzeittest wird ein spezielles Beanspruchungskollektiv zur Reibungsminderung eingesetzt. Dieses senkt die Reibung auf ein Niveau, welches dem der uneingelaufenen Friktionspaarung ähnlich ist. Auf Basis von Untersuchungen zum Schädigungs- und Erholungsverhalten trockenlaufender Friktionspaarungen liegen Erfahrung und Messdaten aus Untersuchungen vor, in welchen die Beanspruchung während der Schädigung und der Erholung variiert wird [14]. Daraus ist bekannt, dass die Reibung nicht nur unter hohen Beanspruchungen absinken kann. Hierzu zählen auch gewisse

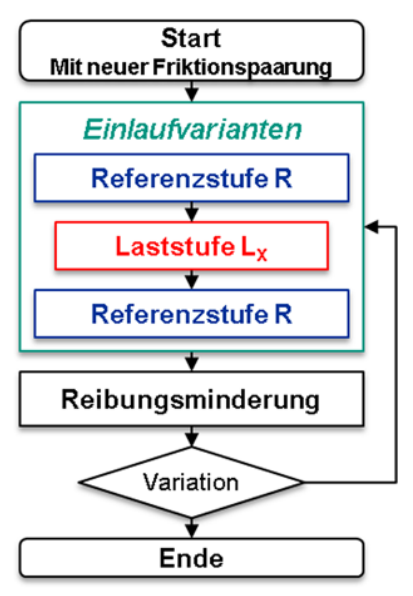

Abb. 2 Versuchsablauf des Kurzzeittest

Bestandteile des Beanspruchungskollektivs wie unter anderem die Richtungsumkehr und ebenso das unvollständige Abbremsen, welche zu Einbrüchen der Reibung von organischen Friktionspaarungen führen können. Der Versuchsablauf ist in Abb. 2 dargestellt.

Innerhalb des Kurzzeittest wird die Friktionspaarung somit immer wieder unter verschiedenen Varianten eingelaufen, welche zu verschiedenen Reibeigenschaften führen. Nach jeder dieser Einlaufvarianten wird die Reibung wieder gemindert. Neben der Variation werden die einzelnen Varianten mehrfach wiederholt. Auf Basis der Ergebnisse wird in kurzer Zeit anhand von vielen verschiedenen Varianten abgeschätzt, welche dieser Varianten zu Verbesserungen des Einlaufverhaltens hinsichtlich des schnellen und sicheren Erreichens der gewünschten Reibeigenschaften führt. Inwieweit dies auch unter den Einlaufbedingungen der realen Anwendung - mit einer neuen Friktionspaarung - gelingt, wird in einem weiteren Versuch überprüft. Hierzu werden zwei weitere Friktionspaarungen dem bisherigen Einlauf sowie der neuen Einlaufvarianten unterzogen, um diese erneut zu vergleichen.

Abb. 3 Trockenreibprüfstand in der Konfiguration mit Schwungmassenmodul [15]

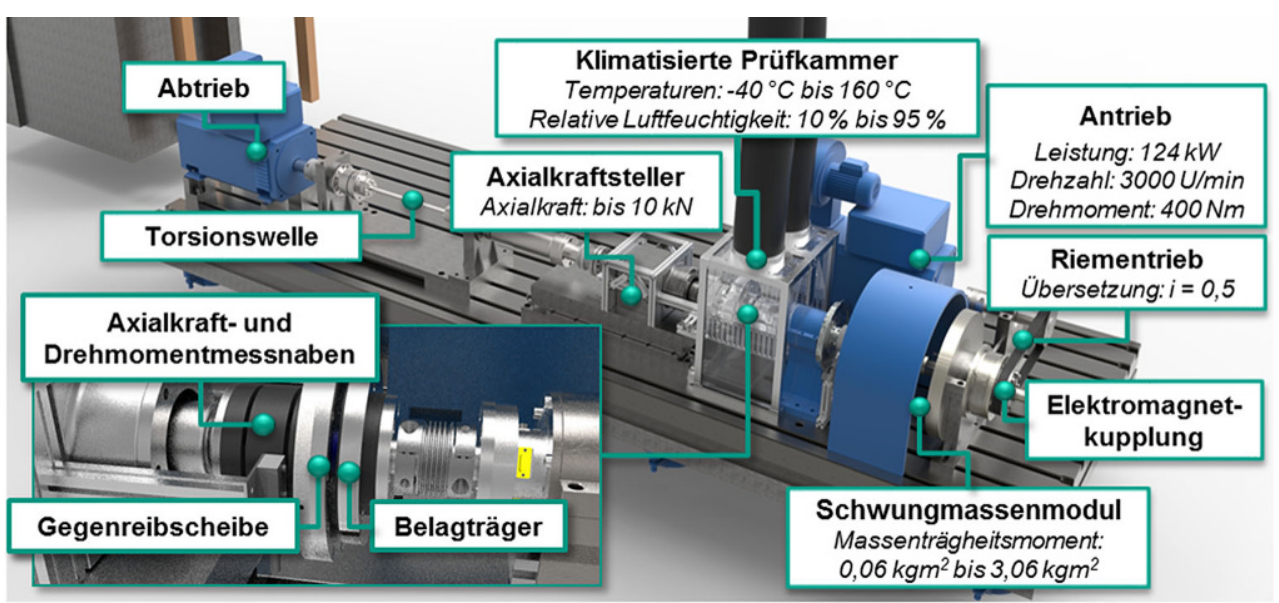


Abb. 4 Darstellung der Messdaten über der Zeit

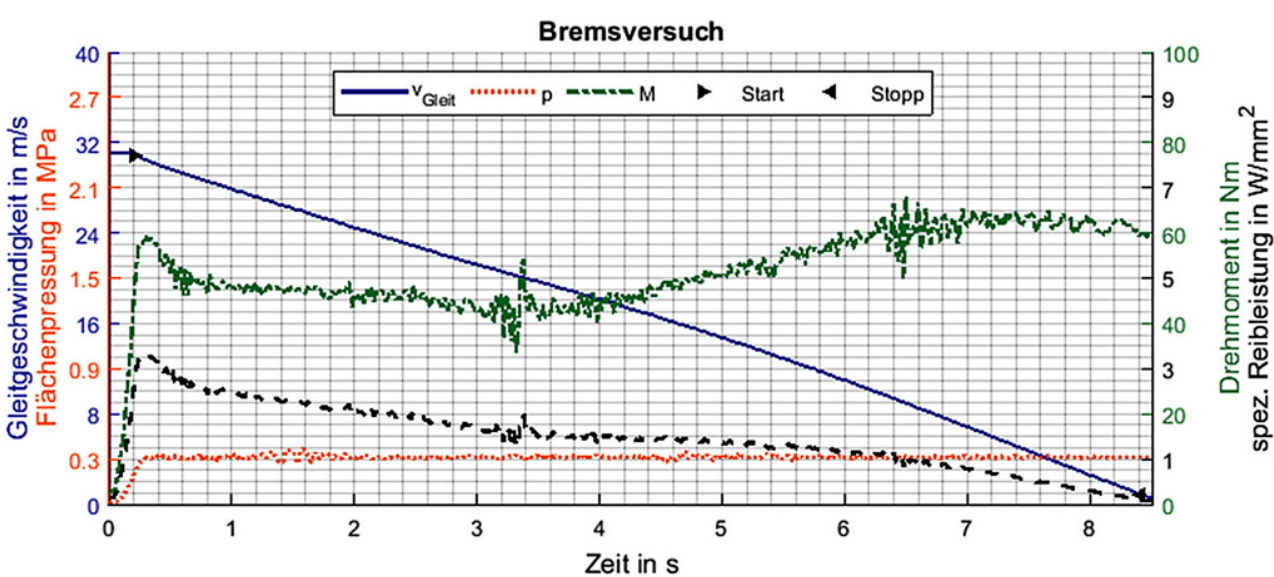

\section{Prüfumgebung: Trockenreibprüfstand}

Für die Untersuchungen wird der TRP - Trockenreibprüfstand des IPEK - Institut für Produktentwicklung in der Konfiguration mit Schwungmassenmodul eingesetzt. Dieser ist in Abb. 3 dargestellt.

Der TRP ermöglicht es, die im Friktionskontakt wirkende thermomechanische Beanspruchung verschiedener Anwendungen und der damit inbegriffenen Wechselwirkungen mit dem Restsystem abzubilden. Der Antrieb realisiert durch die Übersetzung mittels Riementrieb Drehzahlen von bis zu $6000 \mathrm{U} / \mathrm{min}$ an der Schwungmasse. Die Elektromagnetkupplung ermöglicht beim Schalten die Entkopplung der Schwungmasse vom Antrieb. Die nominelle Flächenpressung im Prüfkopf wird über den Axialkraftsteller und die Axialkraftmessnabe geregelt. Die klimatisierte Prüfkammer, welche den Prüfkopf umschließt, dient der Vorgabe konstanter und reproduzierbarer Umgebungsbedingungen. Für die hier gezeigten Versuche wurde eine gleichbleibende Lufttemperatur von $20^{\circ} \mathrm{C}$ und eine relative Luftfeuchtigkeit von $30 \%$ eingestellt. In den Bremsversuchen dieser Arbeit beschleunigt der Antrieb die über den Riementrieb zunächst die Schwungmasse samt dem Belagträger auf die vorgegebene Drehzahl. Anschließend wird die Schwungmasse durch das Öffnen der Elektromagnetkupplung vom Antrieb entkoppelt, bevor der Prüfkopf über den Axialkraftsteller belastet wird. Der Abtrieb des Prüfstands wird für die Bremsversuche nicht benötigt und ist daher verblockt. Der Prüfkopf wird geöffnet, sobald die Antriebsseite vollständig abgebremst wurde. Die nächste Schaltung beginnt, sobald die Messwerte aller in der Gegenreibscheibe integrierten Thermoelementen eine zuvor definierte Starttemperatur unterschreiten. Der Prüfkopf ist im unteren linken Teil von Abb. 3 dargestellt. Die Messnaben zur Erfassung der Axialkraft und des Drehmoments befinden sich auf der Seite des Abtriebs. In der Gegenreibscheibe sind bis zu 16 Thermoelemente radial und zirkular verteilt in Bohrungen eingebracht. Diese befinden sich in Tiefen von
0,2 und $4 \mathrm{~mm}$ unterhalb der Reibfläche. Über diese können Rückschlüsse auf die örtliche Verteilung der thermomechanischen Beanspruchung geschlossen werden [16].

\section{Auswertung der Messdaten}

Die Bremszyklen werden vollautomatisiert ausgewertet. Die Gleitgeschwindigkeit $v_{\text {Gleit }}$ wird dabei aus der Drehzahl $n$ und dem mittleren Reibradius $\mathrm{r}_{\mathrm{m}}$ berechnet.

$v_{\text {Gleit }}=\frac{r_{m} 2 \pi n}{60}$

Die Berechnung des mittleren Reibradius und der nominellen Reibfläche $A_{\text {nom }}$ [17] erfolgen anhand des Innen- und des Außendurchmessers $r_{i}$ und $r_{a}$.

$r_{m}=\frac{2}{3} \frac{r_{a}^{3}-r_{i}^{3}}{r_{a}^{2}-r_{i}^{2}}$

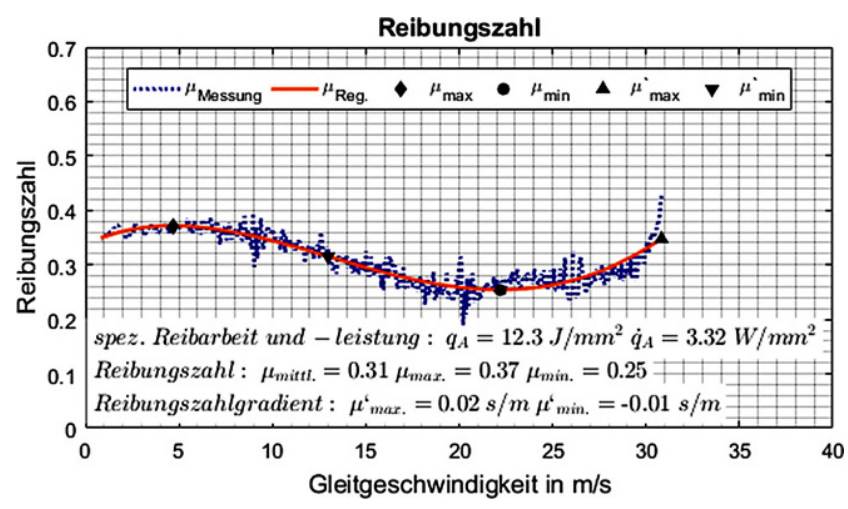

Abb. 5 Darstellung der Reibungszahlen über der Gleitgeschwindigkeit [2] 

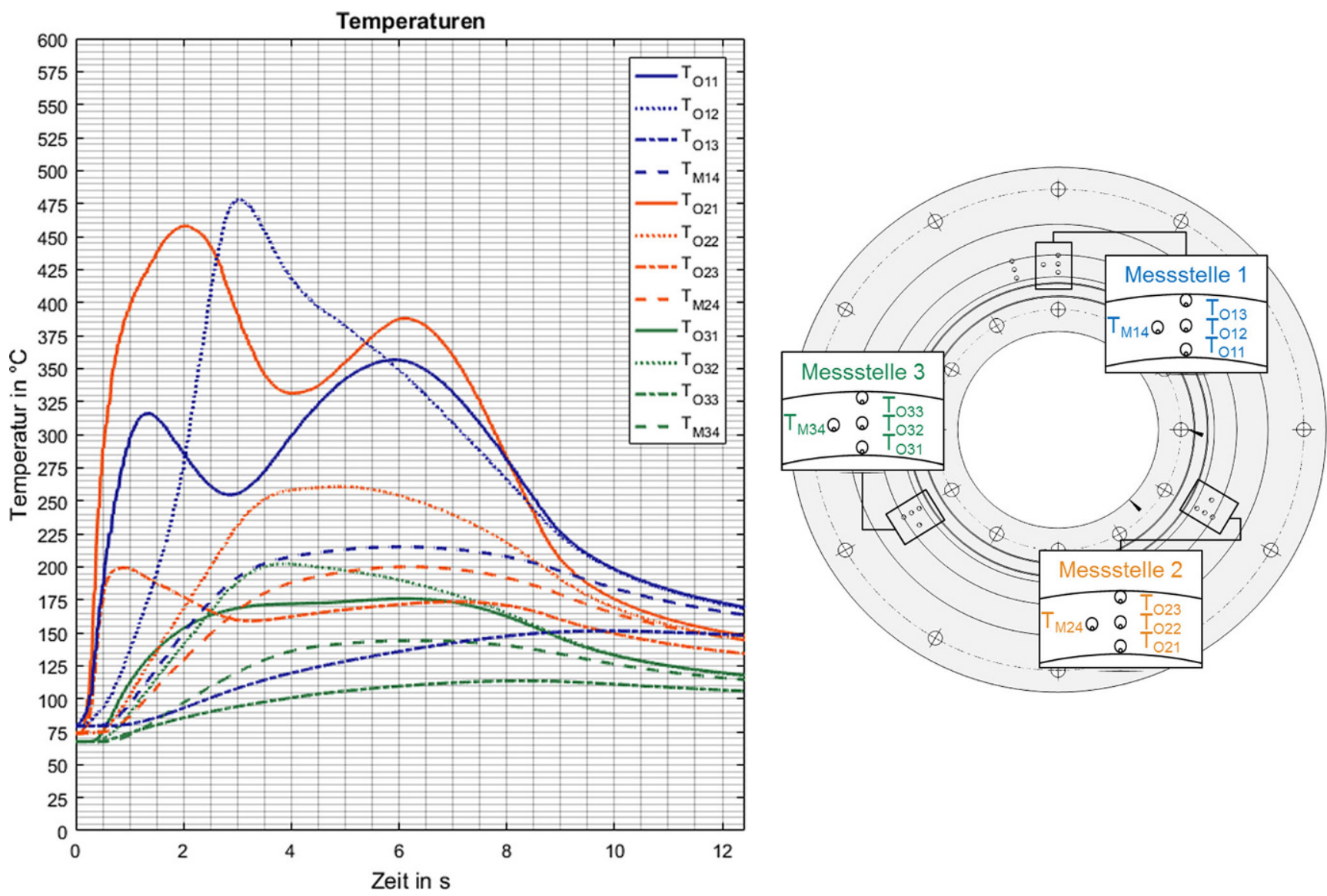

Abb. 6 Temperaturen über der Zeit (links) und deren Positionen in der Gegenreibscheibe; TM in $4 \mathrm{~mm}$ und T0 in 0,2 mm Tiefe zum Reibkontakt (rechts) [2]

Mit der nominellen Reibfläche $\mathrm{A}_{\text {nom }}$ und Normalkraft $\mathrm{F}_{\mathrm{N}}$ wird die nominelle Flächenpressung berechnet.

$p_{\text {nom }=} \frac{F_{N}}{A_{\text {nom }}}$

Die spezifische Reibarbeit wird zur Planung der Versuche vereinfacht mithilfe der Drezahl $\mathrm{n}$, dem Massenträgheitsmoment $\mathbf{J}$ und der nominellen Reibfläche $\mathrm{A}_{\text {nom }}$ berechnet. Bei der Auswertung erfolgt ihre Berechnung durch die Integration der spezifischen Reibleistung über der Zeit.

$q_{A}=\frac{1}{2} \frac{J n^{2}}{A_{\text {nom }}}$

Die spezifische Reibleistung selbst wird mithilfe der nominellen Flächenpressung p pom, der Reibungszahl $\mu$ und Gleitgeschwindigkeit $\mathrm{v}_{\text {Gleit }}$ berechnet.

$\dot{q}_{A}=p_{\text {nom }} \mu v_{\text {Gleit }}$
Die Reibungszahl wird aus der Normalkraft $\mathrm{F}_{\mathrm{N}}$ dem Drehmoment $\mathrm{M}$, dem mittleren Reibradius $\mathrm{r}_{\mathrm{m}}$ und der Anzahl der Reibflächen z berechnet.

$\mu=\frac{M}{F_{N} r_{m} z}$

Aus den Messpunkten zwischen Start und Stopp der Schaltung wird die mittlere Reibungszahl $\mu_{\mathrm{m}}$ als deren Mittelwert gebildet. Diese Messgrößen werden für jeden einzelnen Bremszyklus über der Zeit dargestellt, siehe Abb. 4.

Die Reibungszahlen werden zudem über der Gleitgeschwindigkeit dargestellt, siehe Abb. 5. Hinzu kommt dabei die Regression mittels eines Polynom vierten Grades. Mit diesem und dessen Ableitung über der Gleitgeschwindigkeit werden die Minima und Maxima der Reibungszahl, $\mu_{\min }$ und $\mu_{\max }$, und des Reibungszahlgradienten, $\mu_{\text {min }}$ und $\mu^{\prime}{ }_{\text {max }}$ berechnet.

Weitere Kennwerte sind die Maximalwerte der Temperatursignale für die einzelnen Messstellen und für die gesamte Scheibe. Diese Maxima werden anhand der zeitlichen Verläufe der Messsignale einzelnen beziehungsweise aller Thermoelemente bestimnmt. Die Verläufe der Temperatu- 
Tab. 1 Parameter der Beanspruchungskollektive

\begin{tabular}{lllllll}
\hline Parameter & $\mathrm{T}_{\text {Start }}$ & $\mathrm{p}_{\text {Nom }}$ & $\mathrm{v}_{\text {Gleit }}$ & $\mathrm{q}_{\mathrm{A}}$ & $\mathrm{q}_{\mathrm{A}}$ & tRutsch \\
\hline Einheit & ${ }^{\circ} \mathrm{C}$ & $\mathrm{MPa}$ & $\mathrm{m} / \mathrm{s}$ & $\mathrm{J} / \mathrm{mm}^{2}$ & $\mathrm{~W} / \mathrm{mm}^{2}$ & $\mathrm{~s}$ \\
$\begin{array}{l}\text { Referenz- } \\
\text { stufe }\end{array}$ & 80 & 0,31 & 9 & 1,4 & 1,0 & - \\
Laststufe 1 & 80 & 0,31 & 6 & 0,7 & 0,7 & - \\
Laststufe 2 & 80 & 0,22 & 12 & 2,4 & 1,3 & - \\
Laststufe 3 & 80 & 0,22 & 9 & 1,4 & 0,7 & - \\
Laststufe 4 & 80 & 0,41 & 9 & 1,4 & 1,3 & - \\
$\begin{array}{l}\text { Dauer- } \\
\text { schlupf }\end{array}$ & 80 & 0,31 & -9 & 2,9 & 1,0 & 3 \\
\hline
\end{tabular}

ren der Thermoelemente in der Gegenreibscheibe werden über der Zeit dargestellt, siehe Abb. 6.

\section{Experimentelle Ergebnisse und Diskussion}

Die untersuchte Friktionspaarung besteht aus einem gewickelten, organischen Reibbelag und einer Gegenreibscheibe aus GGG40, Gusseisen mit Kugelgraphit. Die Armierung des Reibbelags besteht aus Glasfasern. Zudem enthält er Füllstoffe wie unter anderem Kupfer, Zink, Eisen, Niob und Schwefel. Kohlenstoff liegt sowohl im Bindemittel als auch in Form von Graphit im Füllstoff vor. Ähnlich wie in einer seiner Anwendungen, in Kupplungsscheiben für trockenlaufenden Anfahrkupplungen von PKW, wird der Reibbelag über Federbleche angebunden. Der Reibbelag wird außerdem auf den Innen- beziehungsweise Außendurchmesser 158 und $182 \mathrm{~mm}$ gedreht. Diese Reduzierung der nominellen Reibfläche hat den Vorteil, dass bei gleichen Kräften höhere nominelle Flächenpressungen gefahren werden können. Das Wandern des mittleren Reibradius wird zudem eingeschränkt, wodurch die Streuung der Reibeigenschaften reduziert wird [18]. Wie in seiner typischen Anwendung verfügt der Reibbelag zudem über Radialnuten.

Gemäß der Methode zur Variation des Beanspruchungskollektivs im Einlauf wird diese Friktionspaarungen unter verschiedenen Beanspruchungskollektiven untersucht. Der Parameter sind in Tab. 1 zusammengefasst.

Abb. 7 zeigt Einläufe des Reibbelags mit der Referenzstufe aus Tab. 1 sowie zwei verschiedenen Gegenreibscheiben. Mit C45, unlegiertem Stahl, werden bereits nach wenigen Bremszyklen mittlere Reibungszahlen von circa 0,4 erreicht. Mit GGG40 steigen die Reibungszahlen dagegen vergleichsweise langsam über mehrere hunderte Bremszyklen hinweg und erreichen Werte von knapp unter 0,3. Folglich liegen Unterschiede im Einlaufverhalten der beiden Friktionspaarungen vor, wenn beide mit der Referenzstufe beansprucht werden. Aus weiteren Untersuchungen zum Schädigungs- und Erholungsverhalten ist zudem be-
Tab. 2 Chemische Zusammensetzung der unbeanspruchten und der beanspruchten Oberfläche der Gegenreibscheibe

\begin{tabular}{lll}
\hline Element & Prozentualer Gewichtsanteil \\
\cline { 2 - 3 } & Unbeansprucht & Beansprucht \\
\hline $\mathrm{C}$ & 16,17 & 14,75 \\
$\mathrm{O}$ & 1,39 & 8,88 \\
$\mathrm{Al}$ & 0,00 & 0,26 \\
$\mathrm{Si}$ & 2,10 & 2,49 \\
$\mathrm{~S}$ & 0,00 & 1,43 \\
$\mathrm{Ca}$ & 0,00 & 0,35 \\
$\mathrm{Fe}$ & 80,34 & 71,17 \\
$\mathrm{Cu}$ & 0,00 & 0,67 \\
\hline
\end{tabular}

kannt, dass die beiden Friktionspaarungen je nach Beanspruchungskollektiv sehr unterschiedliche Reibeigenschaften aufweisen können. Im Folgenden wird die Friktionspaarung mit GGG40 unter Anwendung des Kurzzeittest untersucht, wie das Einlaufverhalten verbessert werden kann.

Abb. 8 zeigt die Reibungszahlen aus jeweils der zweiten der insgesamt vier Wiederholungen aus dem Kurzzeittest. Die Reibungszahlen liegen bei allen vier Varianten zu Beginn, in der Referenzstufe $\mathrm{R}$ bei circa 0,3. Je nach Variante zeigen sich anschließend unter den veränderten Beanspruchungen in den Laststufen $\mathrm{L}_{\mathrm{X}} \mathrm{Zu}$ - und Abnahmen der Reibungszahlen. Zunahmen zeigen sich beispielsweise in der Laststufe $\mathrm{L}_{2}$ mit erhöhter Gleitgeschwindigkeit und in der Laststufe $\mathrm{L}_{3}$ mit abgesenkter Flächenpressungen. Darüber hinaus zeigen sich in der drauffolgenden Referenzstufe $\mathrm{R}$ noch weitere Unterschiede. Hier finden sich ebenso $\mathrm{Zu}$ und Abnahmen der Reibungszahl. Die größten Zunahmen liegen bei den Laststufen $\mathrm{L}_{2}$ mit erhöhter Gleitgeschwindigkeit und in $\mathrm{L}_{4}$ mit erhöhter Flächenpressungen vor.

Der Referenzstufe R folgt nun der Dauerschlupf. Dieser sorgt dafür, dass die Reibungszahlen wieder auf ein Niveau von circa 0,3 fallen. Abb. 9 zeigt beispielhaft einen Messschrieb mit einem solchen Dauerschlupf DS. Während diesem steigt die Reibungszahl deutlich an. Der Vergleich der beiden Referenzstufen $\mathrm{R}$ vor und nach diesem zeigt, dass hierdurch die Reibungszahl gemindert werden kann. Der Einlauf scheint der Friktionspaarung dagegen eine Ausrichtung aufgeprägt zu haben. So erfolgt die Bildung der sekundären Plateaus laut Eriksson [6] beispielsweise nur entgegen der Reibrichtung. Durch die Änderung und den Vorzeichenwechsel der Gleitgeschwindigkeit auf einen Wert von $-9 \mathrm{~m} / \mathrm{s}$, siehe Tab. 1 , könnten sich die zuvor entstandenen sekundären Plateaus bedingt durch die reibungsinduzierte Schubspannung teilweise von den primären Plateaus lösen. Durch die Änderung der Reibrichtung könnten sich des Weiteren die Fasern aufstellen und so die Reibung während des Dauerschlupfs DS erhöhen. Anhand der Analyse der Reibeigenschaften wird deutlich, dass sowohl eine Reibungserhöhung als auch die gewünschte Reibungsmin- 

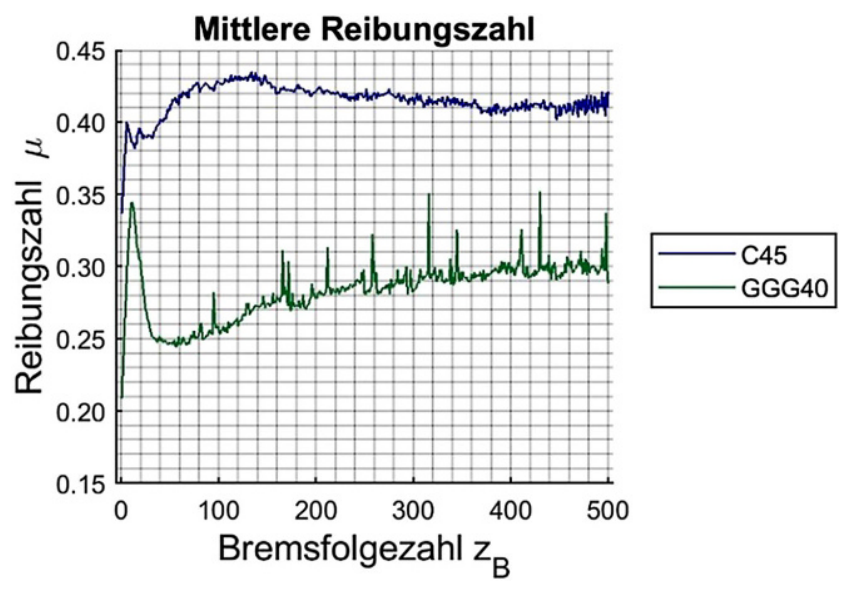

Abb. 7 Vergleich der beiden Friktionspaarungen im Einlauf mit der Referenzstufe derung vorliegen, siehe Abb. 9. Ob diese Effekte tatsächlich durch das Aufstellen der Fasern beziehungsweise das Lösen von sekundären Plateaus verursacht werden, konnte noch nicht nachgewiesen werden und soll in weiterführenden Arbeiten untersucht werden.

Aufgrund der Reibungszunahme, welche bei der Laststufe $\mathrm{L}_{2}$ sowohl in der Laststufe als auch in der darauffolgenden Referenzstufe in allen Wiederholungen beobachtet wurde, wird beschlossen, diese für den Einlauf eines weiteren Exemplars der Friktionspaarung zu verwenden. Die Bremszyklen 25 bis 125 weisen dabei das Beanspruchungskollektiv der Laststufe $\mathrm{L}_{2}$ auf. Alle weiteren Bremszyklen weisen, wie bisher die Parameter der Referenzstufe auf. Abb. 10 zeigt den Vergleich des bisherigen Einlaufs mit dem neuen, optimierten Einlauf. Obwohl nur 100 der 500 Bremszyklen verändert wurden, weist dieser deutlich schneller deutlich höhere mittleren Reibungszahlen von circa 0,41 auf. Dieses Beispiel zeigt somit auf, dass solche Verbesserungen durch vorübergehende Veränderungen des Beanspruchungskollektivs erzielt werden können. Dies wird
Abb. 8 Vergleich der vier Varianten im Kurzzeittest
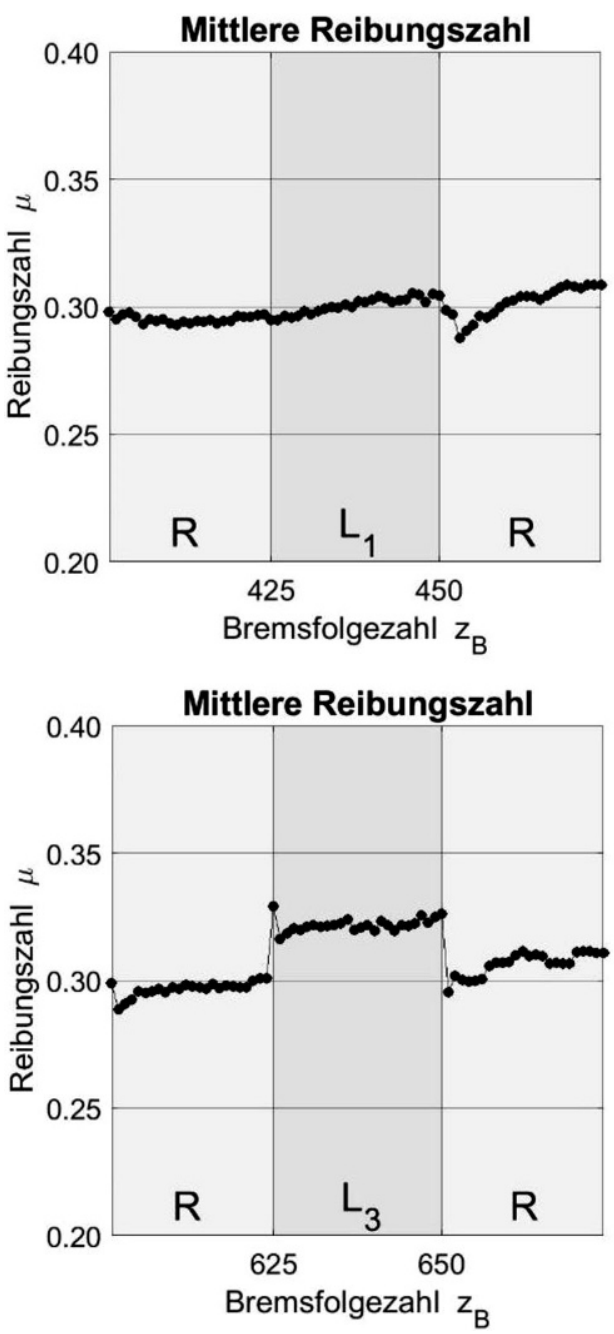
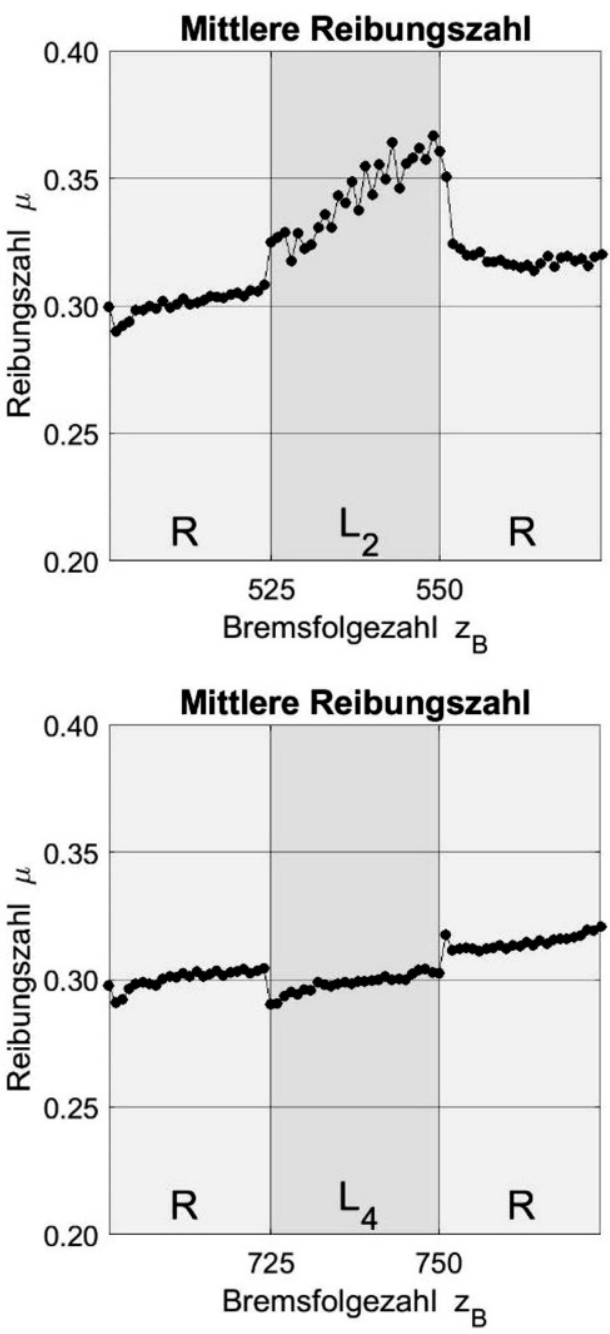


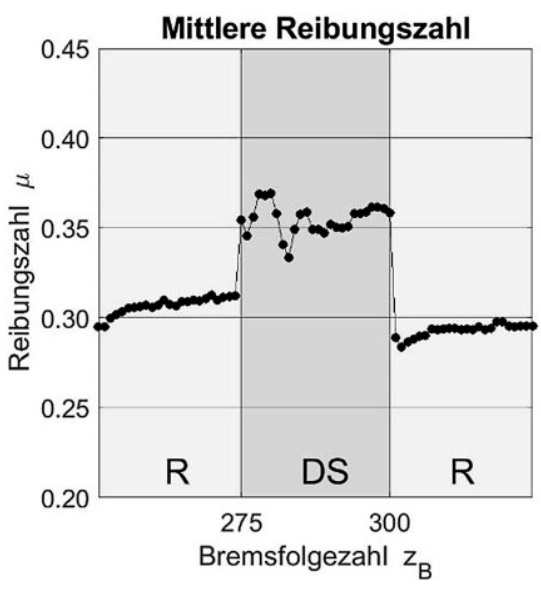

Abb. 9 Reibungsminderung nach abgeschlossenem Dauerschlupf

durch die Anwendung der Methode zur Variation des Beanspruchungskollektivs im Einlauf unterstützt, welche es ermöglicht solche Beanspruchungskollektive in kurzer Zeit zu finden.

Es folgt die Oberflächenanalyse an den Reibflächen der Reibbeläge und der Gegenreibscheiben, um herauszufinden, wie diese Unterschiede zustande kommen. Auf Basis der Untersuchungen zum Schädigungs- und Erholungsverhaltens [14] ist bekannt, dass bei diesem Reibbelag starke Erhöhungen der Beanspruchungen zur Schädigung der Reibschicht und somit zum Einbruch der Reibung führen können. Die glänzende Reibschicht, welche sich im Einlauf ausbildet, löst sich dann von der Reibfläche ab, siehe Abb. 11.

Der Vergleich von Fotoaufnahmen der Reibbeläge nach den Versuchungen zum Einlauf zeigt, dass die Reibbeläge sich nur unwesentlich unterscheiden. Bei allen drei Varian-
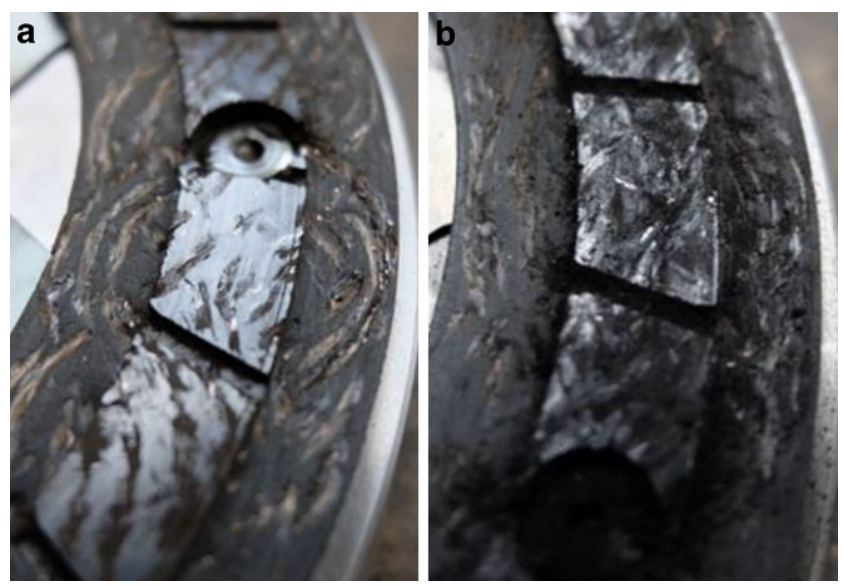

Abb. 11 Fotoaufnahmen der gewickelten Reibbeläge nach (a) dem Einlauf sowie (b) nach Schädigung durch Erhöhung der Gleitgeschwindigkeit

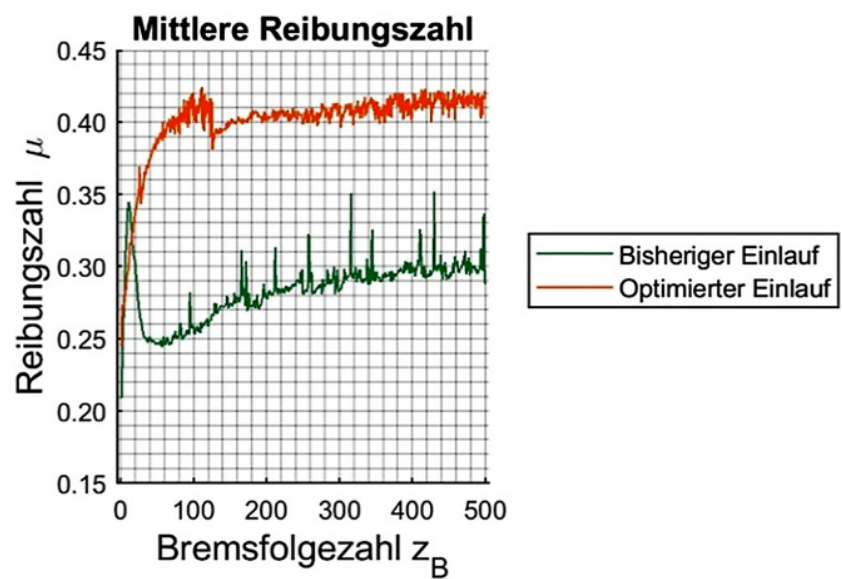

Abb. 10 Vergleich des bisherigen Einlaufs mit der Referenzstufe mit dem optimierten Einlauf mit den 100 Bremszyklen (26 bis 125) bei der Laststufe L2

ten bildet sich bei beiden Werkstoffen und Beanspruchungen die glänzende Reibschicht aus. Diese überdeckt mit Ausnahme der Glasfasern gleichmäßig die gesamte Oberfläche, siehe Abb. 12.

Bei den Fotoaufnahmen der Gegenreibscheiben zeigen sich dagegen große Unterschiede. Während sich die Reibfläche der Gegenreibscheibe aus C45 deutlich von der unbeanspruchten Fläche abhebt, ist die von GGG40 mit dem bisherigen Einlauf nur etwas dunkler aus zuvor. Nach dem optimierten Einlauf fällt außerdem die stärkere bräunliche Färbung der Reibfläche auf. Diese tritt bei der Gegenreibscheibe aus $\mathrm{C} 45$ ebenso auf. Bei derjenigen aus GGG40 mit dem bisherigen Einlauf ist sie jedoch kaum wahrzunehmen, siehe Abb. 13.

Die Proben werden mittels verschiedener Mikroskope und chemischer Analysen untersucht. Abb. 14 und 15 zeigen die Aufnahmen des Lichtmikroskops und des Rasterelektronenmikroskops. Daran sind zum einen die farblichen Unterschiede zu erkennen, welche infolge der Beanspruchung auftreten. Ebenso ändert sich die Struktur der Oberfläche.

Mithilfe der chemischen Analyse durch die energiedispersive Röntgenanalyse werden zudem verschiedene Belagsbestandteile wie unter anderem Schwefel, Aluminium und Kupfer nachgewiesen. Der Vergleich der Ergebnisse mit denen der unbeanspruchten Oberfläche zeigt, dass diese zuvor noch nicht vorhanden sind, siehe Tab. 2 .

Es liegt somit ein Materialübertrag vom Reibbelag hin zur Gegenreibscheibe vor, welcher die farblichen Veränderungen verursacht. Dessen Bildung scheint durch die Steigerung der Beanspruchung in der Laststufe $\mathrm{L}_{2}$ gesteigert zu werden. Auf Basis der Ergebnisse ist davon auszugehen, dass dieser an der Erhöhung der Reibung im optimierten Einlauf beteiligt ist. Die Analogie hierzu ist Gummiabrieb auf einer Rennstrecke, welcher zur Erhöhung der Reibung 

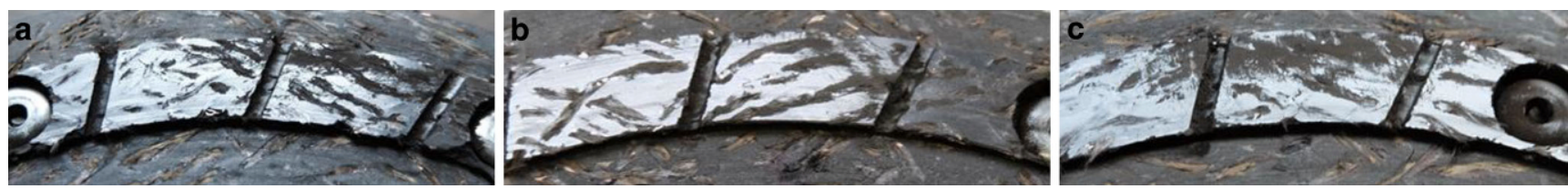

Abb. 12 Fotoaufnahmen der gewickelten Reibbeläge nach a dem Einlauf mit C45, b dem Einlauf mit GGG40 und c dem optimierten Einlauf mit GGG40

Abb. 13 Fotoaufnahmen der Gegenreibscheiben nach a dem Einlauf mit C45, b dem Einlauf mit GGG40 und $\mathbf{c}$ dem optimierten Einlauf mit GGG40
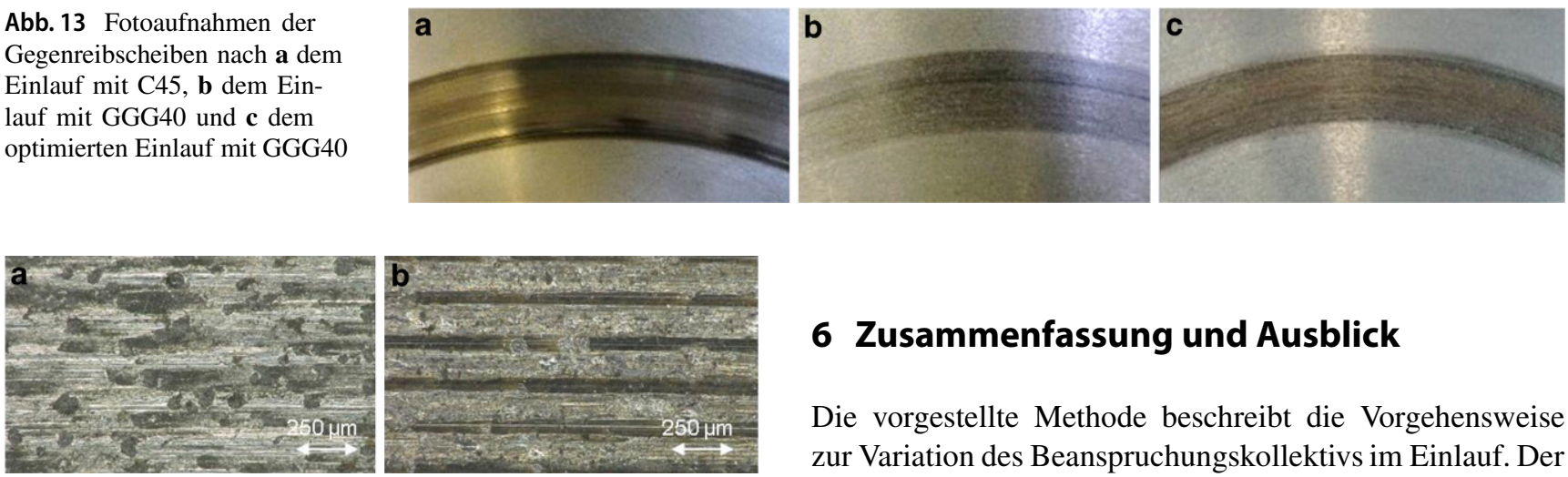

Abb. 14 Lichtmikroskopische Aufnahmen der (a) unbeanspruchten und der (b) beanspruchten Oberfläche der Gegenreibscheibe aus GGG40 nach dem optimierten Einlauf

führen kann. Der Materialübertrag scheint bei der Gegenreibscheibe aus GGG40 unter dem bisherigen Einlauf in geringerem $\mathrm{Ma}$ aufzutreten. Eine Variation der Beanspruchung kann auf Basis der Ergebnisse dazu beitragen, diesen zu fördern und somit die Reibung zu erhöhen. Die Studie zeigt somit anhand von beispielhaften Ergebnissen zu einer Friktionspaarung das Potenzial auf, die gewünschten Reibeigenschaften durch eine Variation der Beanspruchung im Einlauf zu erreichen.

Abb. 15 Rasterelektronenmikroskopische Aufnahmen der (a) unbeanspruchten und der (b) beanspruchten Oberfläche der Gegenreibscheibe aus GGG40 nach dem optimierten Einlauf
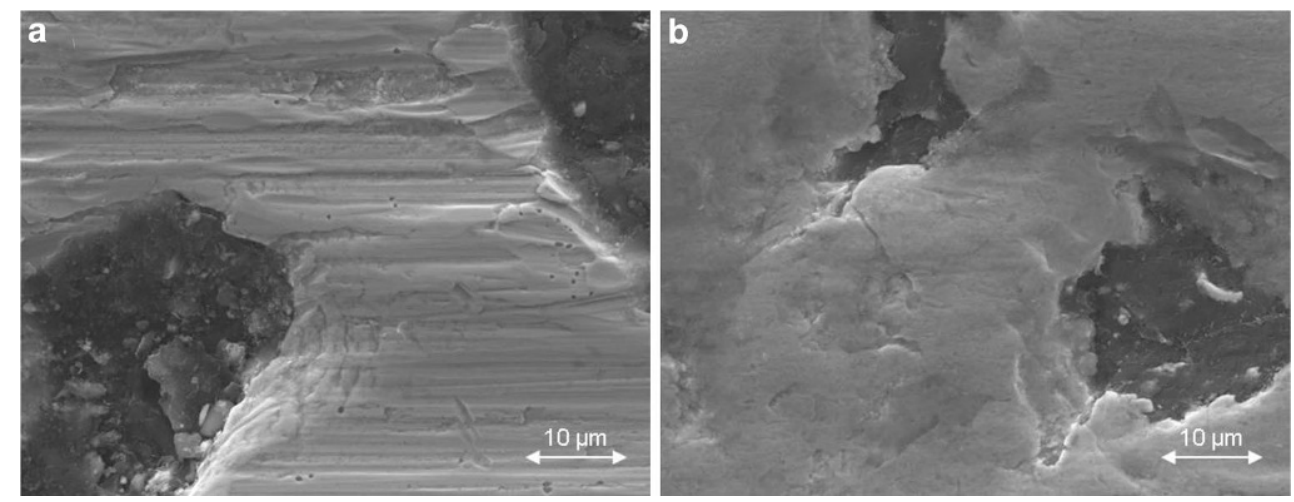
Danksagung Die Autoren danken für die Unterstützung des Forschungsprojekts. Das IGF- Vorhaben 19377-N der Forschungsvereinigung Antriebstechnik e. V. (FVA) wird über die AiF im Rahmen des Programms zur Förderung der Industriellen Gemeinschaftsforschung (IGF) vom Bundesministerium für Wirtschaft und Energie aufgrund eines Beschlusses des Deutschen Bundestages gefördert. Ihr Dank gilt zudem der Firma Schaeffler Automotive Buehl GmbH \& Co. KG für die Unterstützung und die Bereitstellung des Gusswerkstoffs.

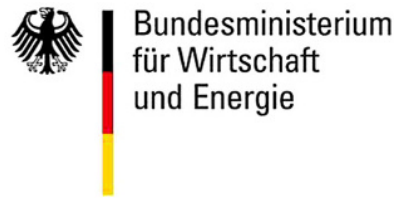

Funding Open Access funding enabled and organized by Projekt DEAL.

Open Access Dieser Artikel wird unter der Creative Commons Namensnennung 4.0 International Lizenz veröffentlicht, welche die Nutzung, Vervielfältigung, Bearbeitung, Verbreitung und Wiedergabe in jeglichem Medium und Format erlaubt, sofern Sie den/die ursprünglichen Autor(en) und die Quelle ordnungsgemäß nennen, einen Link zur Creative Commons Lizenz beifügen und angeben, ob Änderungen vorgenommen wurden.

Die in diesem Artikel enthaltenen Bilder und sonstiges Drittmaterial unterliegen ebenfalls der genannten Creative Commons Lizenz, sofern sich aus der Abbildungslegende nichts anderes ergibt. Sofern das betreffende Material nicht unter der genannten Creative Commons Lizenz steht und die betreffende Handlung nicht nach gesetzlichen Vorschriften erlaubt ist, ist für die oben aufgeführten Weiterverwendungen des Materials die Einwilligung des jeweiligen Rechteinhabers einzuholen.

Weitere Details zur Lizenz entnehmen Sie bitte der Lizenzinformation auf http://creativecommons.org/licenses/by/4.0/deed.de.

\section{Literatur}

1. Klotz T, Bauer T, Ott S, Albers A (2019) Synthese von Beanspruchungskollektiven zur Erholung trockenlau-fender Friktionspaarungen und -systeme. In: Gesellschaft für Tribologie (Hrsg) 60. Tribologie-Fachtagung 2019: Reibung, Schmierung und Verschleiß. Forschung und praktische Anwendungen, S 182-189

2. Klotz T, Ott S, Albers A (2019) Analyse des Schädigungs- und Erholungsverhaltens trockenlaufender Friktionspaarungen. Forsch Ingenieurwes. https://doi.org/10.1007/s10010-019-00307-y
3. Albers A, Ott S, Basiewicz M, Schepanski N, Klotz T (2017) Methode zur Ermittlung der zulässigen thermomechanischen Beanspruchbarkeit trockenlaufender Friktionspaarungen

4. Musiol F (1994) Erklärung der Vorgänge in der Kontaktzone von trockenlaufenden Reibpaarungen über gesetzmäßig auftretende Phänomene im Reibprozeß. Dissertation, TU Berlin, Berlin

5. Völkel K, Pflaum H, Stahl K (2019) Einflüsse der Stahllamelle auf das Einlaufverhalten von Lamellenkupplungen. Forsch Ingenieurwes 83(2):185-197

6. Eriksson M, Jacobson S (2000) Tribological surfaces of organic brake pads. Tribol Int 33(12):817-827

7. Ostermeyer G-P (2002) Dynamik der Reibung in Bremsen. VDIBerichte, Nr. 1736, VDI Verlag, Braunschweig

8. Poeste T (2005) Untersuchungen zu reibungsinduzierten Veränderungen der Mikrostruktur und Eigenspannungen im System Bremse. Dissertation, Berlin

9. Cristol-Bulthé A-L, Desplanques Y, Degallaix G (2007) Coupling between friction physical mechanisms and transient thermal phenomena involved in pad-disc contact during railway braking. Wear 263(7-12):1230-1242

10. Tanaka K, Ueda S, Noguchi N (1973) Fundamental studies on the brake friction of resin-based friction materials. Wear 23(3):349-365

11. Lührsen B (1987) Wirkung der thermischen Belastung auf die Reibpaarung großer Trommel- und Scheibenbremsen

12. Gauger D (1998) Wirkung der thermischen Belastung auf die Reibpaarung großer Trommel- und Scheibenbremsen. Berlin, TU Berlin. Dissertation

13. Fidlin A, Bäuerle S, Boy F (2015) Modelling of the gas induced fading of organic linings in dry clutches. Tribol Int 92:559-566

14. Albers A, Ott S, Klotz T (2020) Kupplungsmodell III: Erholung Trockenlauf : Reib- und Verschleißverhalten während der Erholung trockenlaufender Friktionspaarungen nach thermomechanischer Überlastung. Karlsruhe. Abschlussbericht FVA, Bd. 607 III

15. Klotz T, Ott S, Albers A (2018) Experimentelle Ermittlung und Identifizierung der temporären Schädigungsgrenze trockenlaufender Friktionspaarungen. In: Reibung, Schmierung und Verschleiß: Fachvorträge, wissenschaftliche Poster. Gesellschaft für Tribologie e.V., Aachen

16. Klotz T, Ott S, Albers A (2018) Eine Methode zur Ermittlung der anwendungsspezifischen Leistungsgrenze trockenlaufender Friktionspaarungen. Forsch Ingenieurwes. https://doi.org/10.1007/ s10010-018-0292-y

17. Gesellschaft für Tribologie (2002) GfT Arbeitsblatt 7 Tribologie : Verschleiß, Reibung, Definitionen, Begriffe, Prüfung

18. Albers A, Ott S, Kniel J (2015) Einfluss der Reibringgeometrie auf das tribologische Verhalten. Abschlussbericht FVA, Bd. 607 II 